Applied nutritional investigation

\title{
Dietary patterns among 13-y-old Portuguese adolescents
}

\author{
Joana Araújo M.P.H. ${ }^{\mathrm{a}, \mathrm{b}, *}$, Joana Teixeira M.Sc. ${ }^{\mathrm{a}, \mathrm{b}}$, A. Rita Gaio Ph.D. ${ }^{c, \mathrm{~d}}$, \\ Carla Lopes Ph.D. ${ }^{\mathrm{a}, \mathrm{b}}$, Elisabete Ramos Ph.D. ${ }^{\mathrm{a}, \mathrm{b}}$
}

${ }^{a}$ Department of Clinical Epidemiology, Predictive Medicine and Public Health; and Cardiovascular Research E Development Unit, University of Porto Medical School, Porto, Portugal

${ }^{\mathrm{b}}$ Institute of Public Health of the University of Porto, Porto, Portugal

${ }^{\mathrm{c}}$ Department of Mathematics, Science Faculty, University of Porto, Porto, Portugal

${ }^{\mathrm{d}}$ Centre of Mathematics of the University of Porto, Porto, Portugal

\section{A R T I C L E I N F O}

\section{Article history:}

Received 2 October 2013

Accepted 18 June 2014

\section{Keywords:}

Dietary patterns

Cluster analysis

Diet

Adolescents

\begin{abstract}
A B S T R A C T
Objective: The aim of this study was to identify dietary patterns in 13-y-old adolescents and to evaluate their association with socioeconomic and behavioral factors.

Methods: Data from 1489 adolescents born in 1990 and enrolled in schools of Porto, Portugal, in 2003-2004 (EPITeen study) were analyzed, after excluding those without information on the food frequency questionnaire and the outliers. The questionnaire items were grouped into 14 food groups and dietary patterns were identified by cluster analysis ( $k$-means). Their association with participants' characteristics was computed using multinomial logistic regression.

Results: Four dietary patterns were identified. The pattern healthier (16.1\%) was characterized by the highest consumption of seafood, soup, vegetables/legumes, fruit, and added fats. The dairy products pattern $(29.7 \%)$ showed the highest consumption of dairies. The pattern fast food and sweets (14.2\%) presented the highest intake of fast food, sweets and pastry, soft drinks and coffee or tea. The lower intake pattern (40\%) was characterized by a lower consumption of the majority of food groups. A higher odds of belonging to the fast food and sweets pattern, compared with the lower intake pattern was found among adolescents spending more time watching TV on the weekends (>360 min: odds ratio [OR], 2.09; 95\% confidence interval [CI], 1.23-3.57) and among those consuming four to seven fried meals per week (OR, 3.96; 95\% CI, 2.27-6.90). Adolescents with highly educated parents were less likely to belong to the fast food and sweets group.

Conclusion: Unhealthier behaviors and lower socioeconomic position were the main factors associated with the unhealthier dietary pattern (fast food and sweets). This information should be considered in the development of health-promotion interventions.
\end{abstract}

(c) 2015 Elsevier Inc. All rights reserved.

\section{Introduction}

In nutritional epidemiology the relationship between health and diet has traditionally been examined using the single-food or nutrient approach. However, this approach presents several

JA received grants from the Portuguese Foundation for Science and Technology: POCTI/SAU-ESP/62399/2004, FCOMP-01-0124-FEDER-015750, and SFRH/BD/78153/ 2011. JA supervised data entry and collection of food intake data and drafted the first version of the manuscript. JT and ARG performed the statistical analysis and contributed to the interpretation and discussion of the data. $\mathrm{CL}$ provided significant advice and contributed to the revision and discussion of the manuscript. ER conceived and designed the study, coordinated and supervised data collection, and contributed to the interpretation and discussion of the data. All authors critically reviewed the manuscript and approved the final version. The authors declare that they have no conflict of interest.

* Corresponding author. Tel.: +351 225513 652; fax: +351 225513653 .

E-mail address: jfaraujo@med.up.pt (J. Araújo). limitations related to the inability of taking into account the interactive or synergistic effects of nutrients and foods [1-3].

The study of dietary patterns has been proposed to overcome these issues. This approach has been considered valuable because dietary patterns account for combinations of foods and nutrients and are characterized on the basis of the actual eating behaviors of a specific population [1].

Additionally, the information on dietary patterns of a specific population could be used to develop nutritional or dietary interventions and to create guidelines based on dietary patterns rather than using messages based on separate foods or nutrients as the overall eating patterns might be easier for the public to translate into diets [4].

Two main approaches can be used to study dietary patterns: one denominated a priori and other a posteriori [5,6]. Both methods present advantages and limitations, but the data-driven 
methods (a posteriori approaches) characterize total diet, providing the description of the actual dietary consumption of the population under study $[5,6]$.

Dietary patterns have been mainly studied in adult populations, and previous studies have reported that dietary patterns found in adolescents differ from those in adults $[7,8]$. Additionally, although some of the patterns may be similar between adolescents and adults, the number of patterns found is different between the two age groups [9]. A previous study developed in our population found that some food groups contribute differently to the nutrient intake of adolescents and adults, particularly regarding seafood, sweets, and pastries [10]. This supports differences in diet according to age groups. Additionally, information about dietary intake in adolescents is of crucial importance because changes in diet are expected to occur in this age group. Adolescents are progressively autonomous and their personal choices and preferences gain priority over eating habits of the family [11,12]. For example, sharing fewer meals with the family, such as family dinner, may contribute to these changes [13]. Additionally, some dietary habits are acquired at these ages, making adolescence a potential period for intervention. Previous studies have shown that eating habits, including unhealthy ones, developed during adolescence may track into adulthood if no effective interventions have been outlined [14-16].

Therefore, the aim of this study was to identify and characterize dietary patterns in 13-y-old Portuguese adolescents, using cluster analysis, and to evaluate their association with socioeconomic and behavioral factors.

\section{Methods}

\section{Data collection}

Eligible participants were adolescents born in 1990 and enrolled at public and private schools in Porto, Portugal during the 2003-2004 school year, as part of the EPITeen study [17]. The study was approved by the Ethics Committee of Hospital S. João and procedures were developed to guarantee data confidentiality and protection. Parents and adolescents received written and oral information explaining the purpose and the design of the study and written informed consent was obtained from both.

The evaluation included two self-administered questionnaires (one completed at home, another at school), comprising information on social, demographic, and behavioral characteristics and individual and family history of disease. A physical examination also was performed at school, by a team of experienced nurses, nutritionists, and physicians.

As part of the home questionnaire, food intake was recorded using a food frequency questionnaire (FFQ) regarding the previous $12 \mathrm{mo}$, completed by the adolescents at home with the help of their parents or legal guardians. The previously designed FFQ [18] was adapted for the Portuguese population, as described in detail previously [19]. The questionnaire was validated for the adult population by comparison with $4 \times 7-d$ food records (each one in a different season of the year) $[19,20]$. The FFQ was then adapted for adolescents, including foods more frequently eaten by this age group, and comprised 91 food or beverage items and a frequency section with nine possible responses ranging from never to $\geq 6$ times a day. It also included an open-ended section for foods not listed in the questionnaire, but eaten at least once a week.

Food intake data was obtained by multiplying the frequency of consumption of each food item by the nutrient content of a specified portion size. Seasonal variation of food consumption was also considered according to participants' replies. To estimate nutrient intake from the evaluated food intake, we used the software Food Processor Plus (version 7.2, 1997, ESHA Research, Salem, OR, USA) based on values from the U.S. Department of Agriculture. Values for typical Portuguese foods were added, based on the Portuguese Tables of Food Composition, typical recipes, and data from other studies, as previously described [19, 20]. The nutrient content of the food items that are usually eaten cooked was estimated considering cooking and processing.

We defined 14 food groups (dairy products, seafood, red meat, white meat, pasta/potatoes/rice, cereals, soup, vegetables/legumes, fruit, added fats, fast foods, sweets and pastry, soft drinks and coffee/tea), according to nutritional similarities, as described in Table 1.
Table 1

The 14 food groups considered in the study*

\begin{tabular}{ll}
\hline Food groups & Item \\
\hline Dairy products & Skim, semi-skim, and whole milk; yogurt; cheese \\
Seafood & Fish (included canned), seafood, shellfish, molluscs \\
Red meat & Beef, pork, lamb, viscera, fresh bacon \\
White meat & Chicken, turkey, rabbit \\
Cereals & Bread and breakfast cereals (refined and \\
& whole-grain cereals) \\
Pasta/potatoes/rice & Pasta, potatoes, rice \\
Soup & Vegetable soups \\
Vegetables/legumes & Yellow and green vegetables, salads, beans \\
Fruit & Fresh and canned fruits \\
Added fats & Vegetable oils, butter, margarine \\
Fast foods & Pizza, burgers, sausages, chorizos, meat pies, chips \\
Sweets and pastries & Biscuits, cookies, cakes, pastries, chocolate \\
& preserves, dairy desserts, ice cream \\
Soft drinks & Carbonated and noncarbonated soft drinks \\
Coffee/tea & Coffee, green and black teas \\
\hline
\end{tabular}

* Items aggregated according to nutritional similarities.

Household composition was evaluated asking with whom the adolescent was living and then the variable living with both parents (yes/no) was created. Parental educational level was measured as the number of successfully completed years of formal schooling; we used the information obtained from the parent with the higher education level. We also evaluated parental occupation and classified it as white collar, blue collar, and others (students, housewives, unemployed, and retired). The self-reported weight and height of the parents were evaluated and parents were classified as overweight or obese using body mass index (BMI) and according to the World Health Organization definition [21]. Schools were considered public if they were administered by the state, or private when not administered by local or national government.

Regarding adolescents characteristics, we assessed the frequency of regular practice of sports as the frequency of spending at least 20 min consecutively in sporting activities, beyond compulsory school activities. Additionally, selfperception of leisure-time activities also was evaluated according to four subjective intensity categories (mainly sitting, mainly standing, active, or very active). Adolescents were asked to report the time spent watching television, separately for weekends and weekdays. We used time spent watching TV on the weekends categorized in four categories: $\leq 120,121$ to 240,241 to 360, and $>360$ min.

Some questions about how foods were cooked were also asked. The frequency of consumption of fried meals (such as chips, fried snacks, fried meat, etc.) was assessed using the options: daily, 1 to 3 times, 4 to 6 times, or $<1$ time a week.

Adolescents' weight and height were measured following standardized procedures [22]. Adolescents were classified according to the age- and sexspecific BMI reference percentiles developed by the U.S. Centers for Disease Control and Prevention [23], considering those at or above the 95th percentile as obese and those at or above the 85th percentile but below the 95th percentile as overweight.

\section{Participants}

Among the 2787 eligible participants identified, 44 (1.6\%) could not be reached, $583(20.9 \%)$ refused (no signed informed consent form was returned), and 2160 agreed to participate and provided information at least for part of the planned assessment, resulting in a $77.5 \%$ overall participation, similar in public and private schools (respectively, $77.7 \%$ versus $77 \%$; $P=0.709$ ) [17].

Of the 2160 participants, 247 did not return the home questionnaire, $298 \mathrm{did}$ not complete the FFQ or were excluded as no information was provided on more than $10 \%$ of food items, and 93 participants were not considered because their total energy intake was more than 3 times the interquartile range or their intake of fruit or vegetables was more than 1.5 times the interquartile range. For this specific analysis, an additional 33 adolescents ( 13 girls and 20 boys) were identified as outliers regarding the intake of at least 1 of the 14 food groups considered for the cluster definition, and were removed from the study. The classification of outliers was done empirically, analyzing each empirical food group distribution; at the end, no outlier was below the 99.5th percentile of each distribution. This analysis was thus based on the information of 1489 participants. Compared with the excluded adolescents, the sample included in this analysis presented a higher proportion of girls and a higher proportion of adolescents practicing sports. Additionally, included adolescents were more frequently enrolled at private schools, were more likely to have parents with 
higher levels of education, and more likely to have mothers with white-collar occupations.

\section{Statistical analysis}

Given that significant differences for food groups among boys and girls (univariate analysis of variance) were only found for "cereals" and "coffee/tea," and that a multivariate analysis of variance did not flag a sex affect, dietary patterns were identified with no stratification by sex.

Patterns were identified through the $k$-means algorithm enhanced by bootstrapping and choice of different centers. For each random sampling (with repetitions) of 100 individuals, the $k$-means algorithm with a number of clusters ranging from 1 to 6 was applied to 10 different sets of centers, also obtained randomly from the sample. The final model was the one presenting the lowest sum of squares of the intracluster distances (to the centers).

Different choices of food group units (daily g, percentage of energy [\%energy]) and their treatment (e.g., log-transformed, square-root transformed, adjusted for total energy intake, standardized) were tested as input variables. Among all methodologies, the model that was thought to best represent the nutritional profile of this sample was the one that considered the standardized energy contribution of each food group. These energy contributions were obtained from the linear regression of total energy intake into the 14 food groups, with systematic component given by.

Total Enery $=\beta_{0}+\beta_{1} F G_{1}+\ldots+\beta_{14} F G_{14}$

where $F G_{1}, \ldots, F G_{14}$ denote the 14 food groups.

To compare the characteristics between included and not included participants, and to compare participants and family characteristics according to dietary patterns, we used the $\chi^{2}$ test. Analysis of variance and Tukey's multiple comparisons were used to compare mean intake of food groups or nutrients across the dietary clusters. A multinomial logistic regression model was also computed, considering the lower intake dietary pattern as the reference class because it was the most prevalent group. Odds ratios (OR), and respective $95 \%$ confidence intervals (95\% CI), were computed, controlling for regular practice of sports and parental education.

The software Statistical Package for the Social Sciences (IBM Corp. Released 2011. IBM SPSS Statistics for Windows, Version 20.0. Armonk, NY: IBM Corp.), and the $\mathrm{R}$ language and software environment for statistical computation (version 2.12.1, R Foundation for Statistic Computing, Austria, 2010) were used. The significance level was fixed at 0.05 .

\section{Results}

Four dietary patterns were identified and are characterized in Table 2. The pattern named healthier, which included 239 (16.1\%) participants, was characterized by a higher consumption of seafood [mean $(S D)=93.9(46.7) \mathrm{g} / \mathrm{d}]$, soup [376 (190.8) g/d], vegetables/legumes [338.4 (140.8) g/d], fruit [427.8 (229.2) g/d], and added fats [21.4 (14.5) g/d], in comparison with the other patterns. The 442 adolescents (29.7\%) included in the dairy products group did not present any food group that stands for high or low consumption, in comparison with the other patterns, with the exception of a higher intake of dairy products [650.6 $(338.3) \mathrm{g} / \mathrm{d}]$. There were 212 adolescents $(14.2 \%)$ included in the fast food and sweets pattern; this group was distinguished by the higher intake of fast foods [164.2 (95.6) $\mathrm{g} / \mathrm{d}$ ], sweets and pastries [194.0 (88.8) g/d], soft drinks [671.8 (448.2) g/d], and coffee/tea [40.1 (62.6) g/d]. Finally, the lower intake group included 596 participants $(40 \%)$ characterized by lower consumption of the majority of food groups (dairy products, seafood, red and white meat, cereals, pasta/potatoes/rice, soup, fruit, and added fats) (Table 2). Regarding energy intake (Table 2), adolescents from the fast food and sweets group presented higher total energy intake [3343.2 (482.1) kcal/d] and those from the lower intake group had lower energy intake [1811.9 (378.3) kcal/d]. Considering the intake of macronutrients as the proportion of their contribution to the total energy intake (Table 2), the pattern fast food and sweets was distinguished by a significantly lower intake of protein [15.2 (2.4)\%energy], and the dairy products pattern by a significantly lower intake of total fat [31.1 (4.0) \%energy].

The characterization of adolescents according to the four dietary patterns is presented in Table 3. No differences between dietary patterns were found either by $\operatorname{sex}(P=0.077)$ or by selfclassification of leisure-time activities $(P=0.100)$. A higher proportion of adolescents referring to a higher frequency of regular practice of sports and a lower proportion of those spending more time watching TV belonged to the healthier group. Consumption of fried meals was more frequent among adolescents from the fast food and sweets group and less frequent among those from the healthier pattern. With regard to BMI, a higher proportion of obese adolescents (BMI $\geq 95^{\text {th }}$ percentile) were included in the pattern lower intake in comparison with the other patterns. Regarding social and family characteristics, compared with the other patterns, the healthier and the dairy products groups presented a higher proportion of adolescents

Table 2

Mean (SD) consumption of each food group, and mean energy and nutrient intake, according to dietary pattern

\begin{tabular}{|c|c|c|c|c|}
\hline & Healthier $(\mathrm{n}=239 ; 16.1 \%)$ & Dairy products $(\mathrm{n}=442 ; 29.7 \%)$ & Fast food and sweets $(\mathrm{n}=212 ; 14.2 \%)$ & Lower intake $(n=596 ; 40 \%)$ \\
\hline Food groups $(\mathrm{g} / \mathrm{d})$ & Mean (SD) & & & \\
\hline Dairy products & $538.3(270.4)$ & $650.6(338.3)$ & $532.5(310.6)$ & $404.5(250.7)^{*}$ \\
\hline Seafood & $93.9(46.7)$ & $67.5(33.7)$ & $78.3(48.4)$ & $49.4(28.7)^{*}$ \\
\hline Red meat & $65.3(34.2)$ & $70.8(32.7)$ & $73.0(36.4)$ & $46.6(28.9)^{*}$ \\
\hline White meat & $43.3(29.3)$ & $50.1(27.8)$ & $50.2(29.7)$ & $34.8(22.5)^{*}$ \\
\hline Eggs & $15.6(9.4)$ & $14.4(9.1)$ & $15.4(10.2)$ & $11.2(8.6)^{*}$ \\
\hline Cereals & $118.8(58.3)$ & $142.8(60.0)$ & $135.8(68.9)$ & $70.2(43.3)^{*}$ \\
\hline Pasta/potatoes/rice & $193.6(79.7)$ & $188.1(69.8)$ & $168.2(76.9)$ & $120.8(47.6)^{*}$ \\
\hline Soup & $376.0(190.8)$ & $260.4(192.7)$ & $185.0(163.6)$ & $137.0(140.0)^{*}$ \\
\hline Vegetables/legumes & $338.4(140.8)$ & $108.9(76.1)$ & $160.2(116.1)$ & $104.7(84.5)^{*}$ \\
\hline Fruit & $427.8(229.2)$ & $302.3(165.8)$ & $373.6(232.6)$ & $208.3(135.2)^{*}$ \\
\hline Added fats & $21.4(14.5)$ & $11.9(8.7)$ & $14.9(11.5)$ & $8.4(7.0)^{*}$ \\
\hline Fast foods & $62.6(38.1)$ & $73.1(38.0)$ & $164.2(95.6)$ & $68.3(37.7)^{*}$ \\
\hline Sweets and pastries & $76.5(45.7)$ & $91.9(52.6)$ & $194.0(88.8)$ & $74.8(53.0)^{*}$ \\
\hline Soft drinks & $247.5(207.7)$ & $283.0(214.8)$ & $671.8(448.2)$ & $262.4(232.2)^{*}$ \\
\hline Coffee/tea & $18.5(40.7)$ & $12.2(25.2)$ & $40.1(62.6)$ & $16.8(37.1)^{*}$ \\
\hline Nutrients & Mean (SD) & & & \\
\hline Energy (kcal/d) & $2724.4(487.5)$ & $2621.3(362.3)$ & $3443.2(482.1)$ & $1811.9(378.3)^{*}$ \\
\hline Protein (\%energy) & $17.4(2.4)$ & $17.6(2.3)$ & $15.2(2.4)$ & $17.5(3.0)^{*}$ \\
\hline Carbohydrate (\%energy) & $52.0(5.8)$ & $52.8(5.2)$ & $53.9(6.1)$ & $51.9(6.2)^{*}$ \\
\hline Total fat (\%energy) & $32.7(5.2)$ & $31.1(4.0)$ & $32.8(4.8)$ & $32.2(4.5)^{*}$ \\
\hline
\end{tabular}

Values of foods/nutrients that present a statistically significant higher intake than each of the remaining clusters are printed in bold

Comparisons were performed using Tukey's post hoc tests

* Values of foods/nutrients that present the lower intake 
Table 3

Distribution of dietary patterns identified according to adolescent/family characteristics

\begin{tabular}{|c|c|c|c|c|c|}
\hline & Healthier & Dairy products & Fast food and sweets & Lower intake & $P$-value \\
\hline & \multicolumn{4}{|l|}{$\mathrm{n}(\%)$} & \\
\hline \multicolumn{6}{|l|}{ Sex } \\
\hline Girls & $128(53.6)$ & $217(49.1)$ & $124(58.5)$ & $333(55.9)$ & 0.077 \\
\hline Boys & $111(46.4)$ & $225(50.9)$ & $88(41.5)$ & $263(44.1)$ & \\
\hline \multicolumn{6}{|c|}{ Regular practice of sports } \\
\hline Never & $32(14.4)$ & $65(15.3)$ & $42(22.1)$ & $126(22.3)$ & \\
\hline$\leq 1$ time/wk & $41(18.5)$ & $92(21.7)$ & $41(21.6)$ & $143(25.4)$ & \\
\hline $2-3$ times/wk & $74(33.3)$ & $154(36.3)$ & $60(31.6)$ & $165(29.3)$ & \\
\hline$>3$ times/wk & $75(33.8)$ & $113(26.7)$ & $47(24.7)$ & $130(23.0)$ & 0.003 \\
\hline \multicolumn{6}{|l|}{ Leisure-time activities } \\
\hline Mainly sitting & $58(26.4)$ & $129(30.1)$ & $48(24.1)$ & $177(31.2)$ & 0.100 \\
\hline Mainly standing & $47(21.4)$ & $86(20.1)$ & $49(24.6)$ & $144(25.4)$ & \\
\hline Active/very active & $115(52.3)$ & $213(49.8)$ & $102(51.3)$ & $247(43.5)$ & \\
\hline \multicolumn{6}{|c|}{ TV watching on weekend (min) } \\
\hline$\leq 120$ & $59(25.3)$ & $82(19.2)$ & $26(13.2)$ & $130(22.5)$ & \\
\hline $121-240$ & $91(39.1)$ & $168(39.3)$ & $59(29.9)$ & $177(30.7)$ & \\
\hline $241-360$ & $45(19.3)$ & $99(23.2)$ & $54(27.4)$ & $144(25.0)$ & \\
\hline$>360$ & $38(16.3)$ & $78(18.3)$ & $58(29.4)$ & $126(21.8)$ & $<0.001$ \\
\hline \multicolumn{6}{|c|}{ Frequency of fried meal consumption } \\
\hline$<1$ time/wk & $87(36.9)$ & $109(25.0)$ & $29(13.9)$ & $167(28.6)$ & \\
\hline $1-3$ times/wk & $125(53.0)$ & $270(61.9)$ & $135(64.9)$ & $352(60.4)$ & \\
\hline 4-7 times/wk & $24(10.2)$ & $57(13.1)$ & $44(21.2)$ & $64(11.0)$ & $<0.001$ \\
\hline \multicolumn{6}{|l|}{ BMI* $^{*}$} \\
\hline$<85^{\text {th }}$ & $179(77.8)$ & $327(75.9)$ & $160(81.2)$ & $390(68.7)$ & 0.002 \\
\hline$\geq 85^{\text {th }} \&<95^{\text {th }}$ & $31(13.5)$ & $72(16.7)$ & $24(12.2)$ & $102(18.0)$ & \\
\hline$\geq 95^{\text {th }}$ & $20(8.7)$ & $32(7.4)$ & $13(6.6)$ & $76(13.4)$ & \\
\hline \multicolumn{6}{|l|}{ School } \\
\hline Public & 160 (66.9) & $315(71.3)$ & $172(81.1)$ & $439(73.7)$ & 0.006 \\
\hline Private & $79(33.1)$ & $127(28.7)$ & 40 (18.9) & $157(26.3)$ & \\
\hline \multicolumn{6}{|c|}{ Living with both parents } \\
\hline No & $41(18.5)$ & $89(20.8)$ & $44(22.6)$ & $127(22.2)$ & \\
\hline Yes & $181(81.5)$ & $339(79.2)$ & $151(77.4)$ & $444(77.8)$ & 0.656 \\
\hline \multicolumn{6}{|l|}{ Parental education (y) } \\
\hline $0-6$ & $23(9.7)$ & $74(16.9)$ & $69(33.2)$ & $161(27.2)$ & $<0.001$ \\
\hline $7-9$ & $34(14.3)$ & $79(18.0)$ & $57(27.4)$ & $131(22.2)$ & \\
\hline $10-12$ & $75(31.6)$ & $116(26.4)$ & $51(24.5)$ & $173(29.3)$ & \\
\hline$>12$ & $105(44.3)$ & $170(38.7)$ & $31(14.9)$ & $126(21.3)$ & \\
\hline \multicolumn{6}{|l|}{ Mother's occupation } \\
\hline Blue collar & $22(9.8)$ & 77 (17.9) & $60(29.9)$ & $136(24.1)$ & \\
\hline White collar & $165(73.7)$ & $281(65.5)$ & $87(43.3)$ & $320(56.6)$ & \\
\hline Other $^{\dagger}$ & $37(16.5)$ & $71(16.6)$ & $54(26.9)$ & $109(19.3)$ & $<0.001$ \\
\hline \multicolumn{6}{|l|}{ Mother's BMI $\left(\mathrm{kg} / \mathrm{m}^{2}\right)$} \\
\hline$<25$ & $160(70.5)$ & $281(66.3)$ & $116(59.5)$ & $306(54.4)$ & \\
\hline $25-29.9$ & $47(20.7)$ & $110(25.9)$ & $57(29.2)$ & $174(30.9)$ & \\
\hline$\geq 30$ & $20(8.8)$ & $33(7.8)$ & $22(11.3)$ & $83(14.7)$ & $<0.001$ \\
\hline
\end{tabular}

BMI, body mass index

* According to U.S. Centers for Disease Control and Prevention [23].

Others include students, housewives, unemployed, and retired individuals.

enrolled at private schools, which had parents with higher education level and mainly from white-collar occupations. The proportion of adolescents with mothers reporting a BMI $\geq 30 \mathrm{~kg} /$ $\mathrm{m}^{2}$ was higher among those in the fast food and sweets and in the lower intake groups.

A multinomial logistic regression model was computed to estimate the association between adolescents' and parents' characteristics and dietary patterns, adjusted for regular practice of sports and parental education (Table 4). After adjustment and using the lower intake dietary pattern as reference, adolescents with higher frequency of sports practice presented higher odds of being in the healthier pattern (OR, 1.85; 95\% CI, 1.13-3.03) or in the dairy products pattern (OR, 1.49; $95 \% \mathrm{CI}, 1.00-2.22$ ), whereas those spending more time watching TV on the weekend were about twice as likely to belong to the fast food and sweets group. Adolescents reporting a higher frequency of consuming fried meals were 3.96 times (95\% CI, 2.27-6.90) more likely to be in the fast food and sweets group. Adolescents' BMI was inversely associated with inclusion in the dairy products and fast food and sweets groups. Higher parental education increased the odds of being included in the healthier or the dairy products groups, whereas it decreased the odds of being included in the fast food and sweets group. Adolescents whose mothers were overweight were less likely to belong to the healthier group (OR, 0.66; $95 \% \mathrm{CI}$, $0.45-0.97$ ), and those with obese mothers were less likely to belong to the dairy products group (OR, 0.54; 95\% CI, 0.34-0.84).

\section{Discussion}

In this population-based sample of 13-y-old boys and girls, we identified four dietary patterns: healthier, dairy products, fast food and sweets, and lower intake. The comparison of patterns defined a posteriori in a population with those defined in other studies is difficult due to differences in the methodology used to evaluate food intake and to define dietary patterns. To our knowledge, only few studies $[7,24,25]$ have used cluster analysis to define dietary patterns in this age group. Additionally, eight other studies [9,26-32] explored this a posteriori approach 
Table 4

Multinomial logistic regression model for association between adolescent/family characteristics and dietary patterns

\begin{tabular}{|c|c|c|c|c|c|c|}
\hline & \multicolumn{2}{|l|}{ Healthier } & \multicolumn{2}{|l|}{ Dairy products } & \multicolumn{2}{|c|}{ Fast foods and sweets } \\
\hline & OR $(95 \% \mathrm{CI})^{\dagger}$ & OR $(95 \% \mathrm{CI})$ & OR $(95 \% \mathrm{CI})^{\dagger}$ & OR $(95 \% \mathrm{CI})^{\ddagger}$ & OR $(95 \% \mathrm{CI})^{\dagger}$ & OR $(95 \% \mathrm{CI})$ \\
\hline \multicolumn{7}{|l|}{ Sex } \\
\hline Girls & 1 & 1 & 1 & 1 & 1 & 1 \\
\hline Boys & $1.10(0.81-1.48)$ & $0.85(0.61-1.18)$ & $1.31(1.03-1.68)$ & $1.19(0.91-1.55)$ & $0.90(0.65-1.23)$ & $0.83(0.59-1.17)$ \\
\hline \multicolumn{7}{|c|}{ Regular practice of sports } \\
\hline Never & 1 & 1 & 1 & 1 & 1 & 1 \\
\hline$\leq 1$ times/wk & $1.13(0.67-1.90)$ & $1.04(0.62-1.78)$ & $1.25(0.84-1.86)$ & $1.20(0.80-1.79)$ & $0.86(0.53-1.41)$ & $0.89(0.54-1.46)$ \\
\hline $2-3$ times/wk & $1.77(1.10-2.84)$ & $1.42(0.87-2.30)$ & $1.81(1.25-2.62)$ & $1.57(1.08-2.29)$ & $1.09(0.69-1.72)$ & $1.20(0.75-1.90)$ \\
\hline$>3$ times/wk & $2.27(1.40-3.68)$ & $1.85(1.13-3.03)$ & $1.68(1.14-2.49)$ & $1.49(1.00-2.22)$ & $1.08(0.67-1.76)$ & $1.19(0.73-1.94)$ \\
\hline \multicolumn{7}{|c|}{ TV watching on weekends (min) } \\
\hline$\leq 120$ & 1 & 1 & 1 & 1 & 1 & 1 \\
\hline $121-240$ & $1.13(0.76-1.69)$ & $1.29(0.86-1.95)$ & $1.50(1.06-2.13)$ & $1.67(1.17-2.38)$ & $1.67(1.00-2.79)$ & $1.67(0.99-2.80)$ \\
\hline $241-360$ & $0.69(0.44-1.08)$ & $0.94(0.58-1.51)$ & $1.09(0.75-1.59)$ & $1.37(0.93-2.02)$ & $1.88(1.11-3.17)$ & $1.82(1.07-3.10)$ \\
\hline$>360$ & $0.66(0.41-1.07)$ & $0.96(0.59-1.58)$ & $0.98(0.66-1.46)$ & $1.27(0.84-1.92)$ & $2.30(1.36-3.88)$ & $2.09(1.23-3.57)$ \\
\hline \multicolumn{7}{|c|}{ Frequency of fried food consumption } \\
\hline$<1$ times/wk & 1 & 1 & 1 & 1 & 1 & 1 \\
\hline 1-3 times/wk & $0.68(0.49-0.95)$ & $0.75(0.53-1.05)$ & $1.18(0.88-1.57)$ & $1.26(0.94-1.69)$ & $2.21(1.42-3.43)$ & $2.21(1.42-3.44)$ \\
\hline 4-7 times/wk & $0.72(0.42-1.23)$ & $0.75(0.43-1.30)$ & $1.36(0.89-2.10)$ & $1.42(0.91-2.20)$ & $3.96(2.28-6.86)$ & $3.96(2.27-6.90)$ \\
\hline \multicolumn{7}{|l|}{ BMI $\left(\mathrm{kg} / \mathrm{m}^{2}\right)^{*}$} \\
\hline$<85^{\text {th }}$ & 1 & 1 & 1 & 1 & 1 & 1 \\
\hline$\geq 85^{\text {th }} \&<95^{\text {th }}$ & $0.66(0.43-1.03)$ & $0.64(0.41-1.01)$ & $0.84(0.60-1.18)$ & $0.82(0.58-1.16)$ & $0.57(0.35-0.93)$ & $0.57(0.35-0.92)$ \\
\hline$\geq 95^{\text {th }}$ & $0.57(0.34-0.97)$ & $0.65(0.38-1.11)$ & $0.50(0.32-0.78)$ & $0.54(0.35-0.85)$ & $0.42(0.22-0.77)$ & $0.41(0.22-0.77)$ \\
\hline \multicolumn{7}{|c|}{ Parental education (y) } \\
\hline $0-6$ & 1 & 1 & 1 & 1 & 1 & 1 \\
\hline $7-9$ & $1.82(1.02-3.24)$ & $1.85(1.04-3.30)$ & $1.31(0.89-1.94)$ & $1.30(0.88-1.93)$ & $1.02(0.67-1.54)$ & $1.02(0.67-1.56)$ \\
\hline $10-12$ & $3.04(1.82-5.07)$ & $2.95(1.76-4.94)$ & $1.46(1.02-2.10)$ & $1.41(0.98-2.03)$ & $0.69(0.45-1.05)$ & $0.68(0.45-1.05)$ \\
\hline$>12$ & 5.83 (3.51-9.69) & $5.50(3.29-9.18)$ & $2.94(2.05-4.20)$ & $2.74(1.91-3.94)$ & $0.57(0.35-0.93)$ & $0.56(0.35-0.92)$ \\
\hline \multicolumn{7}{|c|}{ Mother's BMI $\left(\mathrm{kg} / \mathrm{m}^{2}\right)$} \\
\hline$<25.0$ & 1 & 1 & 1 & 1 & 1 & 1 \\
\hline $25.0-29.9$ & $0.52(0.36-0.75)$ & $0.66(0.45-0.97)$ & $0.69(0.52-0.92)$ & $0.80(0.60-1.08)$ & $0.86(0.60-1.25)$ & $0.78(0.54-1.14)$ \\
\hline$\geq 30.0$ & $0.46(0.27-0.78)$ & $0.67(0.39-1.15)$ & $0.43(0.28-0.67)$ & $0.54(0.34-0.84)$ & $0.70(0.42-1.17)$ & $0.61(0.36-1.04)$ \\
\hline
\end{tabular}

BMI, body mass index

The reference class from the dependent variable is the dietary pattern lower intake; statistically significant results are printed in bold

* According to U.S. Centers for Disease Control and Prevention [23].

Crude.

Adjusted for regular practice of sports and parental education.

among adolescents, however, they used factor analysis. We decided to compare our results with those from all the studies found in adolescents, as a previous study [7] compared cluster and principal component analyses and concluded that results from both methods are comparable. As in our study, studies from other countries $[7,9,25,26,28-31]$ have found a healthy pattern or a pattern closer to the healthy diet in adolescents. Most of the studies [7,9,25-29] reported a pattern similar to our fast food and sweets pattern, characterized by higher intake of high energy-dense foods and often labeled as the unhealthy or Western pattern. However, only in our population was a pattern characterized by higher intake of dairy products found. Other studies $[7,24,26,28]$ reported some patterns characterized by a high intake of dairy products among other food groups, but not exclusively characterized by a high intake of these products. In our sample, this pattern was closer to the healthier pattern, namely regarding total energy intake, than to the lower intake and fast food and sweets patterns. Regarding the lower intake pattern found in our study, it was the most prevalent in our sample and it represents adolescents with a generalized lower food intake; adolescents presented the lowest intake of almost all food groups, compared with adolescents from other dietary patterns, and the lowest total energy intake, a median of $1812 \mathrm{kcal} / \mathrm{d}$. This does not mean they have an unhealthy diet; they probably represent the general diet of our sample, which does not stand out by the consumption of any particular food group, either healthy or unhealthy. On the other hand, however, a higher proportion of overweight/obese adolescents were found in this pattern. This result and the lower proportion of overweight/obese adolescents found in the fast food and sweets pattern might be explained by a potential to underreport food consumption, which has been described for obese individuals compared with their normal-weight counterparts [33].

The distribution of dietary patterns in our sample differs according to adolescent and family characteristics. Adolescents reporting higher frequency of fried meals consumption and having parents with lower education levels were more likely to belong to the fast food and sweets group compared with the lower intake group. Additionally, and in accordance with previous results [34], more time spent watching TV on the weekend was found among adolescents from the fast food and sweets group. In Australian adolescents, the Western dietary pattern also was associated with more TV viewing [9]. Additionally, studies conducted in German [29], Greek [28], and Balearic Islands' adolescents [26] reported more time spent watching TV or higher screen time among adolescents in unhealthier patterns, such as those labeled Western or junk food. The HELENA (Healthy Lifestyle in Europe by Nutrition in Adolescence) study, presenting data from European adolescents, also found that more TV viewing was associated with higher adherence to the snacking pattern in boys and to the confectionary and snacking pattern in girls [31]. On the other hand, in Greek adolescents the vegetarian/healthy pattern was positively correlated with sports activity outside school [28], which is in accordance with our results. Regarding the indicators of socioeconomic position, in Australian adolescents the healthy pattern was associated with higher maternal education [9] and in other populations unhealthier patterns were associated with lower socioeconomic 
status (SES) [26,29], as in our study higher parental education level decreased the odds of being in the fast food and sweets group. Previous studies conducted in our sample of adolescents also reported unhealthier food sources of nutrients intake among adolescents from lower SES families [10], and that unhealthier lifestyles tend to aggregate [35].

In our study, dietary intake was evaluated using an FFQ. Although this method of food assessment has some limitations, it has been described as a useful technique for the evaluation of diet in population-based studies, allowing discrimination according to usual energy and nutrient intakes [36]. Our FFQ was developed by our department, based on the FFQ designed by Willett et al [18], and adapted for the adult Portuguese population, as previously described [10]. It was validated for the adult population $[19,20]$, and previous evidence has suggested that for the same population, an FFQ developed for adults could be used in adolescents [37].

We used the $k$-means algorithm for the identification of dietary clusters. Considering different choices of food group units and their treatment, the model that was thought to be more adequate was the one having the set of standardized energy contribution of each food group as input variables. Because the $k$ means algorithm is sensitive to outliers and it might affect the formation of the clusters, the use of a standardized variable (\% energy) is preferable [38]. Other studies have used principal component analysis. In our case, however, the first obtained components from principal component analysis explained a small percentage of the total variance $(<50 \%$, regardless of the choice of the food group units), which was consider too low to provide robustness to the analysis.

In the multinomial logistic regression model, we decided not to include all the variables used in univariate analysis because some are highly correlated. For example, type of school, mother's occupation, and parental education are all indicators of SES and retrieved similar results when we studied its distribution according to dietary patterns. Thus, we decided only to include parental education in the regression model. Regarding sports activity, the variable of the frequency of regular practice of sports, which was more informative than the yes or no question about sports, was included in the final model. Regarding adjustments, after testing the potential confounders, regular practice of sports and parental education were the only variables that showed a relevant effect for the final model.

One limitation of this study is the exclusion of some participants with missing information on diet. These exclusions led to an underrepresentation of adolescents with lower SES. However, it was possible to show the effect of SES in the definition of dietary patterns, which supports that these exclusions did not greatly affect the results of our study. On the other hand, our study was conducted on a large sample of population-based adolescents, with compulsory school education and a high proportion of participation, supporting the external validity of our results.

\section{Conclusions}

About half of adolescents were classified in the healthier or dairy products groups, which are characterized by a food intake closer to a healthy diet. The pattern characterized by higher consumption of fast food, sweets, and soft drinks is associated with longer time spent in sedentary activities and with lower SES. Therefore, interventions promoting healthier dietary habits should take into account the information here described.

\section{References}

[1] Hu FB. Dietary pattern analysis: a new direction in nutritional epidemiology. Curr Opin Lipidol 2002;13:3-9.

[2] van Dam RM. New approaches to the study of dietary patterns. Br J Nutr 2005;93:573-4.

[3] Jacobs DR Jr, Steffen LM. Nutrients, foods, and dietary patterns as exposures in research: A framework for food synergy. Am J Clin Nutr 2003;78(3 Suppl):508S-13S.

[4] National Research Council-Comittee on Diet and Health. Diet and health: implications for reducing chronic disease risk. Washington, DC: National Academy Press; 1989.

[5] Moeller SM, Reedy J, Millen AE, Dixon LB, Newby PK, Tucker KL, et al. Dietary patterns: challenges and opportunities in dietary patterns research an experimental biology workshop, April 1, 2006. J Am Diet Assoc 2007; 107:1233-9.

[6] Tucker KL. Dietary patterns, approaches, and multicultural perspective. Appl Physiol Nutr Metab 2010;35:211-8.

[7] Hearty AP, Gibney MJ. Dietary patterns in Irish adolescents: a comparison of cluster and principal component analyses. Public Health Nutr; 2011: $1-10$.

[8] Cutler GJ, Flood A, Hannan P, Neumark-Sztainer D. Major patterns of dietary intake in adolescents and their stability over time. J Nutr 2009;139:323-8.

[9] Ambrosini GL, Oddy WH, Robinson M, O'Sullivan TA, Hands BP, de Klerk $\mathrm{NH}$, et al. Adolescent dietary patterns are associated with lifestyle and family psycho-social factors. Public Health Nutr 2009;12:1807-15.

[10] Araujo J, Severo M, Lopes C, Ramos E. Food sources of nutrients among 13year-old Portuguese adolescents. Public Health Nutr 2011;14:1970-8.

[11] Shepherd R, Dennison CM. Influences on adolescent food choice. Proc Nutr Soc 1996;55:345-57.

[12] Thomas J. Food choices and preferences of schoolchildren. Proc Nutr Soc 1991;50:49-57.

[13] Gillman MW, Rifas-Shiman SL, Frazier AL, Rockett HR, Camargo CA Jr, Field AE, et al. Family dinner and diet quality among older children and adolescents. Arch Fam Med 2000;9:235-40.

[14] Lien N, Lytle LA, Klepp KI. Stability in consumption of fruit, vegetables, and sugary foods in a cohort from age 14 to age 21. Prev Med 2001;33:217-26.

[15] Mikkila V, Rasanen L, Raitakari OT, Pietinen P, Viikari J. Consistent dietary patterns identified from childhood to adulthood: the cardiovascular risk in young Finns study. Br J Nutr 2005;9:923-31.

[16] Li J, Wang Y. Tracking of dietary intake patterns is associated with baseline characteristics of urban low-income African-American adolescents. J Nutr 2008;138:94-100.

[17] Ramos E, Barros H. Family and school determinants of overweight in 13 year-old Portuguese adolescents. Acta Paediatr 2007;96:281-6.

[18] Willett WC, Sampson L, Stampfer MJ, Rosner B, Bain C, Witschi J, et al. Reproducibility and validity of a semiquantitative food frequency questionnaire. Am J Epidemiol 1985;122:51-65.

[19] Lopes C. Reproducibility and validity of semiquantitative food frequency questionnaire. In: Diet and myocardial infarction: a community-based case-control study [PhD Thesis]. Porto: University of Porto; 2000.

[20] Lopes C, Aro A, Azevedo A, Ramos E, Barros H. Intake and adipose tissue composition of fatty acids and risk of myocardial infarction in a male Portuguese community sample. J Am Diet Assoc 2007;107:276-86.

[21] Clinical guidelines on the identification, evaluation, and treatment of overweight and obesity in adults: Executive summary. Expert Panel on the identification, evaluation, and treatment of overweight in adults. Am J Clin Nutr 1998;68:899-917.

[22] World Health Organization. Measuring obesity: classification and description of anthropometric data. Report on a WHO consultation on the epidemiology of obesity (EUR/ICP/NUT 125). Copenhagen: WHO; 1988.

[23] Kuczmarski RJ, Ogden CL, Guo SS, Grummer-Strawn LM, Flegal KM, Mei Z, et al. 2000 CDC growth charts for the United States: methods and development. Vital Health Stat 11; 2002:1-190.

[24] Grieger JA, Scott J, Cobiac L. Cluster analysis and food group consumption in a national sample of Australian girls. J Hum Nutr Diet 2012;25:75-86.

[25] Ritchie LD, Spector P, Stevens MJ, Schmidt MM, Schreiber GB, StriegelMoore RH, et al. Dietary patterns in adolescence are related to adiposity in young adulthood in black and white females. J Nutr 2007;137:399-406.

[26] Bibiloni Mdel M, Martinez E, Llull R, Pons A, Tur JA. Western and Mediterranean dietary patterns among Balearic Islands' adolescents: socioeconomic and lifestyle determinants. Public Health Nutr 2012;15:683-92.

[27] Cutler GJ, Flood A, Hannan P, Neumark-Sztainer D. Multiple sociodemographic and socioenvironmental characteristics are correlated with major patterns of dietary intake in adolescents. J Am Diet Assoc 2011;111:230-40.

[28] Kourlaba G, Panagiotakos DB, Mihas K, Alevizos A, Marayiannis K, Mariolis A, et al. Dietary patterns in relation to socio-economic and lifestyle characteristics among Greek adolescents: a multivariate analysis. Public Health Nutr 2009;12:1366-72.

[29] Richter A, Heidemann C, Schulze MB, Roosen J, Thiele S, Mensink GB. Dietary patterns of adolescents in Germany-associations with nutrient intake and other health related lifestyle characteristics. BMC Pediatr 2012;12:35. 
[30] de Moraes AC, Adami F, Falcao MC. Understanding the correlates of adolescents' dietary intake patterns. A multivariate analysis. Appetite 2012;58:1057-62.

[31] Santaliestra-Pasias AM, Mouratidou T, Huybrechts I, Beghin L, CuencaGarcia M, Castillo MJ, et al. Increased sedentary behaviour is associated with unhealthy dietary patterns in European adolescents participating in the HELENA study. Eur J Clin Nutr 2014;68:300-8.

[32] Rahmawaty S, Lyons T, Wall P, Batterham M, Charlton K, Meyer BJ. Food patterns of Australian children ages 9 to $13 \mathrm{y}$ in relation to omega to 3 long chain polyunsaturated intake. Nutrition 2014;30:169-76.

[33] Bandini LG, Schoeller DA, Cyr HN, Dietz WH. Validity of reported energy intake in obese and nonobese adolescents. Am J Clin Nutr 1990;52:421-5.
[34] Ramos E, Costa A, Araujo J, Severo M, Lopes C. Effect of television viewing on food and nutrient intake among adolescents. Nutrition 2013;29:1362-7.

[35] Fraga S, Severo M, Costa D, Lopes C, Ramos E. Clustering behaviours among 13-year-old Portuguese adolescents. J Public Health 2011;(19 Suppl 1):S21-7.

[36] Araujo MC, Yokoo EM, Pereira RA. Validation and calibration of a semiquantitative food frequency questionnaire designed for adolescents. J Am Diet Assoc 2010;110:1170-7.

[37] Shatenstein B, Amre D, Jabbour M, Feguery H. Examining the relative validity of an adult food frequency questionnaire in children and adolescents. J Pediatr Gastroenterol Nutr 2010;51:645-52.

[38] Newby PK, Tucker KL. Empirically derived eating patterns using factor or cluster analysis: A review. Nutr Rev 2004;62:177-203. 